\title{
The shape of the Hanle curve in spin-transport structures in the presence of the ac drive
}

\author{
R. C. Roundy ${ }^{1}$, M. C. Prestgard ${ }^{2}$, A. Tiwari ${ }^{2}$, and M. E. Raikh ${ }^{1}$ \\ ${ }^{1}$ Department of Physics and Astronomy, University of Utah, Salt Lake City, UT 84112, USA \\ ${ }^{2}$ Department of Materials Science and Engineering, \\ University of Utah, Salt Lake City, Utah 84112, USA
}

\begin{abstract}
Resistance between two ferromagnetic electrodes coupled to a normal channel depends on their relative magnetizations. The spin-dependent component, $R$, of the resistance changes with magnetic field, $B$, normal to the directions of magnetizations. In the field of spin transport, this change, $R(B)$, originating from the Larmour spin precession, is called the Hanle curve. We demonstrate that the shape of the Hanle curve evolves upon application of an ac drive and study this evolution theoretically as a function of the amplitude, $B_{1}$, and frequency, $\omega$, of the drive. If the distance between the electrodes, $L$, is smaller than the spin-diffusion length, $\lambda_{s}$, the prime effect of a weak circular-polarized drive is the shift of the center of the curve to the value of $B$ for which the Larmour frequency, $\omega_{L}$, is $\sim B_{1}^{2} / \omega$. Magnetic resonance at $\omega_{L} \sim \omega$ manifests itself in the derivative, $\frac{d R}{d B}$. For large $L \gg \lambda_{s}$ the ac drive affects the Hanle curve if the drive amplitude exceeds the spin relaxation rate, $\tau_{s}^{-1}$, i.e. at $B_{1} \tau_{s} \gtrsim 1$. The prime effect of the drive is the elimination of a minimum in $R(B)$. Linearly polarized drive has a fundamentally different effect on the Hanle curve, affecting not its shape, but rather its width.
\end{abstract}

PACS numbers: 72.15.Rn, 72.25.Dc, 75.40.Gb, 73.50.-h, 85.75.-d

\section{INTRODUCTION}

In the past decade there has been remarkable progress in the fabrication of lateral F-N-F structures, see Fig. 1. which exhibit spin transport. In the pioneering experiment Ref. 1 the existence of spin transport in an $\mathrm{Al}$ strip was demonstrated by measuring a voltage, $V$, generated between the strip and Co electrode upon injecting a current, $I$, through the other Co electrode. The sign
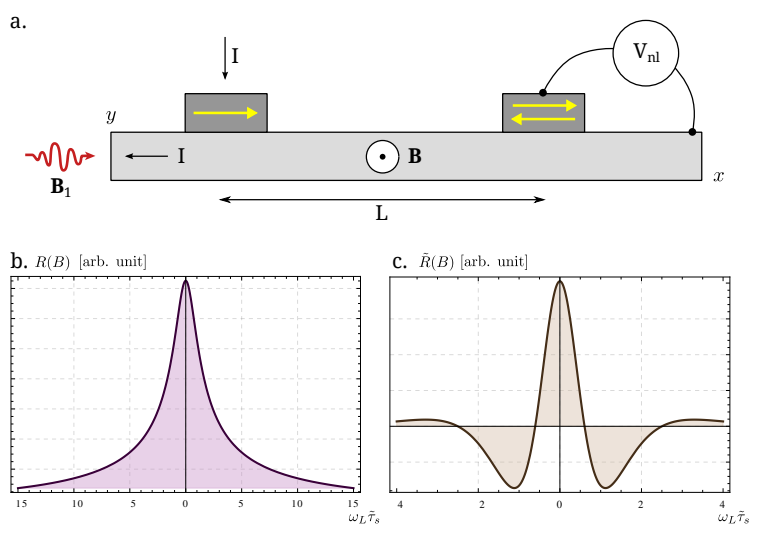

FIG. 1: [Color online] (a) Schematics of a standard spintransport device. Nonlocal resistance is defined as a voltage between the channel and the right ferromagnet detector generated upon injecting the current through the left ferromagnetic electrode. While the polarized electrons travel diffusively the distance, $L$, their spin precesses in magnetic field, $B$, directed along the $z$-axis. The driving ac field, $B_{1}$ is either circularly polarized in the $x-y$-plane or linearly polarized along $x$. The shape of the Hanle curves in (b) short $L / \lambda_{s}=0.1$ and (c) long, $L / \lambda_{s}=2.4$, devices are calculated from Eq. (5). of voltage could be reversed upon reversal of the relative magnetizations of the electrodes. Quantitative information about the spin transport was inferred from the dependence of the generated voltage on a weak external field, $B$, which caused the spin precession. In particular, it was observed that for the average spin precession angle $180^{\circ}$ the generated voltage changes the sign.

A theory for the $\frac{V}{I}=R(B)$ dependence, i.e. for the Hanle profile, was first developed in Refs. 2, 3. Following Ref. 1, a concise derivation of the analytical result of Refs. 2, 3 goes as follows. Suppose that the magnetizations of the injector and detector are directed along the $x$-axis, while the field, $B$, is directed along the $z$ axis. After a time, $t$, from the moment of injection the average $x$-projection of spin of a given electron is $S_{x}(t)=e^{-t / \tau_{s}} \cos \omega_{L} t$, where $\omega_{L}=\gamma B$ is the Larmour frequency ( $\gamma$ is the gyromagnetic ratio), and $\tau_{s}$ is the spin-flip time in the nonmagnetic material. If the motion of electron between the electrodes is a 1D drift, then the times of arrival to the detector are distributed as $P(t)=\frac{1}{\sqrt{4 \pi D t}} \exp \left[-\left(L-v_{d} t\right)^{2} / 4 D t\right]$. Here $L$ is the distance between the electrodes, see Fig. 1 $v_{d}$ is the drift velocity, and $D$ is the diffusion coefficient. Then the nonlocal resistance, $R(B)$, is proportional $S_{x}(t)$ weighted with the distribution $P(t)$, i.e.

$$
R(B)=R_{0} \int_{0}^{\infty} \frac{d t \cos \left(\omega_{L} t\right)}{\sqrt{4 \pi D t}} \exp \left[-\frac{t}{\tau_{s}}-\frac{\left(L-v_{d} t\right)^{2}}{4 D t}\right] .
$$

The prefactor $R_{0}$ is $B$-independent and is proportional to the product of polarizations of the injector and detector.

The integral Eq. (1) contains four parameters of the device: $D, \tau_{s}, L$, and $v_{d}$. In fact, the $B$ dependence of $R$ is governed by only two dimensionless combinations: 
$\omega_{L} \tilde{\tau}_{s}$, where $\tilde{\tau}_{s}$ is the renormalized spin-flip time

$$
\tilde{\tau}_{s}=\frac{\tau_{s}}{1+\frac{v_{0}^{2} \tau_{s}}{4 D}},
$$

and the dimensionless length

$$
\tilde{L}=\frac{L}{\left(4 D \tilde{\tau}_{s}\right)^{1 / 2}} .
$$

Besides, the integral can be evaluated analytically 117 , and expressed in terms of the function $f(y)$ defined as

$$
f(y)=\int_{0}^{\infty} \frac{d s}{s^{1 / 2}} \exp \left[-\frac{1}{s}-y s\right]=\left(\frac{\pi}{y}\right)^{1 / 2} \exp \left[-2 y^{1 / 2}\right] .
$$

Then the $B$-dependence of the nonlocal resistance is simply given by

$$
\begin{aligned}
& R(B) \propto f_{r}(y)=\operatorname{Ref}(y) \\
= & \left(\frac{\pi}{|y|}\right)^{1 / 2} \exp \left[-2|y|^{1 / 2} \cos \frac{\phi}{2}\right] \cos \left(\frac{\phi}{2}+2|y|^{1 / 2} \sin \frac{\phi}{2}\right),
\end{aligned}
$$

where the absolute value, $|y|$, and the phase, $\phi$, of the complex argument, $y$, are defined as

$$
|y|=\tilde{L}^{2}\left(1+\omega_{L}^{2} \tilde{\tau}_{s}^{2}\right)^{1 / 2}, \quad \phi=\arctan \left(\omega_{L} \tilde{\tau}_{s}\right) .
$$

It follows from Eq. (5) that there are two characteristic shapes of the Hanle curve, loosely speaking, shortdevice shape and long-device shape. They are illustrated in Fig. 1.

With regard to experiments, Eq. (1) provides a remarkably accurate description of the Hanle curves measured in various spin-transport devices. Both shapes of $R(B)$ have been reported in many papers, see e.g. Refs. 4423. Usually the value $\tau_{s}$ is inferred from $R(B)$, since $R(B)$ falls off at values $\omega_{L} \sim \tau_{s}^{-1}$. For example, in silicon-based $\frac{448}{8}$ and germanium-based $\frac{910}{10}$ structures the widths of the Hanle curves are $\sim 5 \mathrm{mT}$, so that the values of $\tau_{s}$ is long, $\tau_{s} \sim 5$ ns. Equally, in GaAs the Hanle curves are narrow $\frac{11}{15}$ with similar widths. On the other hand, in graphene-based valves $\frac{16}{20}$ the Hanle curves are broad with widths $\sim 100 \mathrm{mT}$. In InGaAs 21221 the widths are intermediate $\sim 20 \mathrm{mT}$.

It should be noted that determination of both $\tau_{s}$ and the spin-diffusion length, $\lambda_{s}=\left(D \tau_{s}\right)^{1 / 2}$, from a single measured Hanle curve is somewhat ambiguous, in the sense, that the same $R(B)$ can be very well fitted with two significantly different sets of $\tau_{s}$ and $\lambda_{s}$. To improve the accuracy of determination of these parameters in Ref. 12 the Hanle curves for several values of $L$ were analyzed.

Overall, the excellent agreement of the experimentally measured Hanle profiles with theoretical prediction
Eq. (5) seems surprising, since the theory is based on a rather crude description of the spin dynamics of injected carriers. For example, this description completely neglects the details of injection, such as geometry of electrodes. Modeling the transport as a purely 1D diffusion is also somewhat questionable ${ }^{24}$. On the other hand, a complete understanding of the domain of applicability and limitations of the drift-diffusion theory of spin transport seems crucial, since the contemporary research on inverse spin Hall effect $\frac{13|21 / 25| 28}{28}$ and its possible applications in the logic devices contains the driftdiffusion description at its core. One way of testing the drift-diffusion theory, which have already been realized experimentally 29130, is to operate with spatially inhomogeneous spin-density profiles. For example in Ref. 30 this profile was created using the interference of two laser beams.

In the present manuscript we suggest another "knob" to test the drift-diffusion theory. Namely, we demonstrate that the Hanle profile can be manipulated by the ac drive. More specifically, we assume that, in addition to a static field $B_{0}$, an ac field in the $x-y$ plane is applied. On general grounds, one can expect that the ac drive suppresses the $R(B)$ response by affecting the steady precession $S_{x}(t)=\cos \omega_{L} t$. It is also apparent that the drive should make the most pronounced effect on $R(B)$ if the drive frequency, $\omega$, is comparable to $\tilde{\omega}_{L}$ - the value corresponding to the width of the Hanle curve in the absence of drive. For $\omega \gg \tilde{\omega}_{L}$ the ac field oscillates many times as an electron travels between the injector and detector, so that the effect of a weak drive with amplitude $\gamma B_{1} \ll \omega$ averages out. It is somewhat unexpected that, in addition to a simple broadening, the drive gives rise to specific features in the shape of the Hanle curves.

Below we find and analyze the expression for $R(B)$ for ac field with arbitrary amplitude and frequency for the case when it is circularly polarized. The prime effect is the shift of the center of $R(B)$ to the left or to the right depending whether the polarization of the drive is left or right. We also analyze the evolution of the Hanle curves with increasing drive for the case when the drive is linearly polarized. In particular, we identify two peculiar regimes of the spin dynamics which are specific to linear polarization. They are realized when the drive is either very fast or very strong. We discuss how this dynamics manifests itself in the Hanle profile.

\section{DYNAMICS OF THE LARMOUR SPIN PRECESSION IN THE PRESENCE OF THE AC DRIVE}

To find the shape of the Hanle curve in the presence of the ac drive, $\boldsymbol{B}_{1}(t)$, it is necessary to solve the equation for the spin dynamics

$$
\frac{d \boldsymbol{S}}{d t}+\gamma\left(\boldsymbol{B}+\boldsymbol{B}_{1}(t)\right) \times \boldsymbol{S}=0
$$


with initial conditions $S_{x}(0)=1, S_{y}(0)=S_{z}(0)=0$. Then the solution should be substituted into Eq. (1) instead of $\cos \omega_{L} t$. We assume that external field is directed along $z$, i.e. $\boldsymbol{B}=B_{0} \boldsymbol{k}$, while for the ac field lies in the $x-y$ - plane. For this field we will consider the cases of circular and linear polarization separately.

\section{A. Circular polarization}

It is important that the components of the ac drive

$$
B_{x}=B_{1} \cos (\omega t+\varphi) \quad B_{y}=B_{1} \sin (\omega t+\varphi)
$$

contain a random initial phase, $\varphi$. It emerges as a result of the randomness of the time moments at which electrons are injected from the electrode. The nonlocal resistance should be averaged over this phase.

For circular polarization the dynamics of the spin components can be found exactly, since in the rotating frame the ac field is static. We reproduce this textbook solution to track the random phase, $\varphi$, which leads to averaging out of certain contributions to $R(B)$.

In the rotating frame, $x^{\prime}=x \cos \omega t+y \sin \omega t, y^{\prime}=$ $y \cos \omega t-x \sin \omega t$, the general solution of the Bloch equation has the Rabi form

$$
\begin{aligned}
\boldsymbol{S}^{\prime}(t)=\left(\boldsymbol{S}_{0}-\right. & \left.\frac{\left(\boldsymbol{H} \cdot \boldsymbol{S}_{0}\right) \boldsymbol{H}}{H^{2}}\right) \cos \gamma H t \\
& +\frac{\boldsymbol{H} \times \boldsymbol{S}_{0}}{H} \sin \gamma H t+\frac{\left(\boldsymbol{H} \cdot \boldsymbol{S}_{0}\right) \boldsymbol{H}}{H^{2}},
\end{aligned}
$$

where the projections of the vector $\boldsymbol{H}$, which is the effective magnetic field in the rotating frame, are defined as $H_{x^{\prime}}=B_{1} \cos \varphi, H_{y^{\prime}}=B_{1} \sin \varphi$, and $H_{z^{\prime}}=B_{0}-\frac{\omega}{\gamma}$. After implementing the initial condition $S_{x^{\prime}}(0)=1$ it is instructive to rewrite Eq. (9) in components

$$
\begin{aligned}
& S_{x^{\prime}}(t)=\left(1-\frac{B_{1}^{2} \cos ^{2} \varphi}{B_{1}^{2}+\left(B_{0}-\frac{\omega}{\gamma}\right)^{2}}\right) \cos \gamma H t+\frac{B_{1}^{2} \cos ^{2} \varphi}{B_{1}^{2}+\left(B_{0}-\frac{\omega}{\gamma}\right)^{2}}, \\
& S_{y^{\prime}}(t)=\frac{B_{1}^{2} \cos \varphi \sin \varphi}{B_{1}^{2}+\left(B_{0}-\frac{\omega}{\gamma}\right)^{2}}(1-\cos \gamma H t)+\frac{B_{0}-\frac{\omega}{\gamma}}{\sqrt{B_{1}^{2}+\left(B_{0}-\frac{\omega}{\gamma}\right)^{2}}} \sin \gamma H t, \\
& S_{z^{\prime}}(t)=-\frac{B_{1}\left(B_{0}-\frac{\omega}{\gamma}\right) \cos \varphi}{B_{1}^{2}+\left(B_{0}-\frac{\omega}{\gamma}\right)^{2}}(1-\cos \gamma H t)-\frac{B_{1} \sin \varphi}{\sqrt{B_{1}^{2}+\left(B_{0}-\frac{\omega}{\gamma}\right)^{2}}} \sin \gamma H t .
\end{aligned}
$$

It is now seen from Eq. 10 that $S_{z^{\prime}}$ vanishes after averaging and so does the first term in $S_{y^{\prime}}$. In the remaining terms the averaging amounts to the replacement of $\cos ^{2} \varphi$ by $1 / 2$.

Going back to the laboratory system: $S_{x}=S_{x^{\prime}} \cos \omega t-$ $S_{y^{\prime}} \sin \omega t, S_{y}=S_{y^{\prime}} \cos \omega t+S_{x^{\prime}} \sin \omega t$, we get

$$
\begin{aligned}
& \left\langle S_{x}(t)\right\rangle=\frac{B_{1}^{2}}{2 H^{2}} \cos \omega t+\frac{1}{2}\left(1-\frac{B_{1}^{2}}{2 H^{2}}+\frac{B_{0}-\frac{\omega}{\gamma}}{H}\right) \cos [(\gamma H+\omega) t]+\frac{1}{2}\left(1-\frac{B_{1}^{2}}{2 H^{2}}-\frac{B_{0}-\frac{\omega}{\gamma}}{H}\right) \cos [(\gamma H-\omega) t] \\
& \left\langle S_{y}(t)\right\rangle=\frac{B_{1}^{2}}{2 H^{2}} \sin \omega t+\frac{1}{2}\left(1-\frac{B_{1}^{2}}{2 H^{2}}+\frac{B_{0}-\frac{\omega}{\gamma}}{H}\right) \sin [(\gamma H+\omega) t]-\frac{1}{2}\left(1-\frac{B_{1}^{2}}{2 H^{2}}-\frac{B_{0}-\frac{\omega}{\gamma}}{H}\right) \sin [(\gamma H-\omega) t] .
\end{aligned}
$$

We see the averaged dynamics of $S_{x}$ and $S_{y}$ represents oscillations with driving frequency, $\omega$, which are modulated by the "Rabi" envelope with frequency

$$
\gamma H=\sqrt{\left(\omega-\gamma B_{0}\right)^{2}+\left(\gamma B_{1}\right)^{2}}
$$

Thus, while the Rabi oscillations in $S_{z}(t)$ do not survive averaging over initial phase, $\varphi$, they are still present in the averaged dynamics of $S_{x}$ and $S_{y}$. In the next section we study how this dynamics manifests itself in nonlocal resistance. 


\section{NONLOCAL RESISTANCE}

Three contributions to $S_{x}$ in Eq. (11) give rise to three terms in the nonlocal resistance, $R(\bar{B})$. It is convenient to express $R(B)$ through the same function, $f_{r}(y)$, which describes the Hanle shape in the absence of drive and is defined by Eq. (4). One finds

$$
\begin{array}{r}
R(B) \propto\left\{\frac{B_{1}^{2}}{2 H^{2}} f_{r}\left(y_{\omega}\right)+\frac{1}{2}\left(1-\frac{B_{1}^{2}}{2 H^{2}}+\frac{B_{0}-\frac{\omega}{\gamma}}{H}\right) f_{r}\left(y_{\omega+\gamma H}\right)\right. \\
\left.\quad+\frac{1}{2}\left(1-\frac{B_{1}^{2}}{2 H^{2}}-\frac{B_{0}-\frac{\omega}{\gamma}}{H}\right) f_{r}\left(y_{-\omega+\gamma H}\right)\right\} . \quad(14)
\end{array}
$$

Here the arguments $y_{\omega}$ and $y_{ \pm \omega+\gamma H}$ are defined by Eq. (6) with $\omega_{L}$ replaced by $\omega$ and $\pm \omega+\gamma H$, respectively. It is convenient to analyze the shape of the Hanle curves for short and long devices separately.

\section{A. Small distance between the electrodes}

In the limit of small $\tilde{L} \ll 1$ the function Eq. 5 for nonlocal resistance in the absence of drive simplifies to

$$
R(B) \propto \frac{\sqrt{\sqrt{1+\omega_{L}^{2} \tilde{\tau}_{s}^{2}}+1}}{\sqrt{1+\omega_{L}^{2} \tilde{\tau}_{s}^{2}}}=\left.\frac{\tilde{L}}{(2 \pi)^{1 / 2}} f_{r}\left(\omega_{L}\right)\right|_{\tilde{L} \ll 1}
$$

Naturally, it contains only a single scale $\omega_{L} \sim \tilde{\tau}_{s}^{-1}$.

If the magnetization of the injector is along the $x$-axis while the magnetization of the detector is along the $y$ axis, then the Hanle signal is proportional to $S_{y}(t)$. The corresponding expression for nonlocal resistance reads

$$
\begin{array}{r}
\tilde{R}(B) \propto\left\{\frac{B_{1}^{2}}{2 H^{2}} f_{i}\left(y_{\omega}\right)+\frac{1}{2}\left(1-\frac{B_{1}^{2}}{2 H^{2}}+\frac{B_{0}-\frac{\omega}{\gamma}}{H}\right) f_{i}\left(y_{\omega+\gamma H}\right)\right. \\
\left.\quad-\frac{1}{2}\left(1-\frac{B_{1}^{2}}{2 H^{2}}-\frac{B_{0}-\frac{\omega}{\gamma}}{H}\right) f_{i}\left(y_{-\omega+\gamma H}\right)\right\}, \quad(16)
\end{array}
$$

where the function $f_{i}(y)$ is defined through Eq. (5) as $\operatorname{Im} f(y)$, which amounts to the change of cosine by sine in the right-hand-side. In the absence of drive, the resistance $\tilde{R}(B)$ is an odd function of magnetic field. In the limit of small $\tilde{L}$ it simplifies to

$$
\tilde{R}(B) \propto \frac{\sqrt{\sqrt{1+\omega_{L}^{2} \tilde{\tau}_{s}^{2}}-1}}{\sqrt{1+\omega_{L}^{2} \tilde{\tau}_{s}^{2}}}=\left.\frac{\tilde{L}}{(2 \pi)^{1 / 2}} f_{i}\left(\omega_{L}\right)\right|_{\tilde{L} \ll 1}
$$

To find the shape of the Hanle curve in the presence of drive, the asymptote Eq. (15) should be substituted into Eq. (14). In Fig. 2 we plot the modified Hanle curves calculated for the driving frequency $\omega \tilde{\tau}_{s}=7$ and two magnitudes of the drive $\gamma B_{1} \tilde{\tau}_{s}=4$ and $\gamma B_{1} \tilde{\tau}_{s}=6$. We also plot the corresponding curves for $\tilde{R}(B)$. The chosen

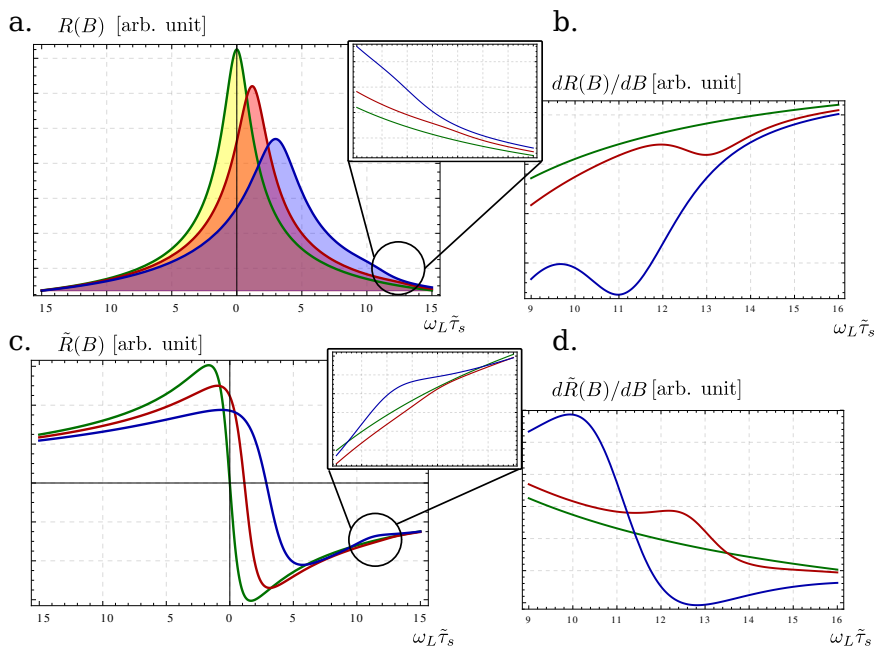

FIG. 2: [Color online] (a) Upon increasing the drive amplitude the Hanle curve broadens and its maximum is shifted quadratically with $B_{1}$. Green, red, and blue curves are plotted from Eq. 14 dimensionless drive frequency $\omega \tilde{\tau}_{s}=7$ and three dimesionless drive amplitudes: $\gamma B_{1} \tilde{\tau_{s}}=0$ (green curve) $\gamma B_{1} \tilde{\tau_{s}}=4$ (red curve), and $\gamma B_{1} \tilde{\tau_{s}}=6$ (blue curve). Magnetic resonance $\omega_{L}=\omega$ resides around $\omega_{L} \tilde{\tau}_{s}=11$. The corresponding region is enlarged. Magnetic resonance is pronounced in the $d R / d B$ shown in (b); (c) and (d) are the same as (a) and (b) for the nonlocal resistance $\tilde{R}(B)$.

value of $\omega$ is so big because the FWHM value of $R(B)$ in the absence of drive is also big, approximately $4 / \tilde{\tau}_{s}$. One can identify in Fig. 2 three major features caused by the drive:

(i) The shift of the maximum. The origin of this shift is the interplay of the prefactor and the function $f_{r}\left(y_{-\omega+\gamma H}\right)$ in the third term of Eq. (14). Firstly, this term gives the dominant contribution to $R(B)$ for small $B_{1}$. This is because the prefactor in the first term is $\propto B_{1}^{2}$, while the prefactor in the second term is $\propto B_{1}^{4}$ for $\omega_{L}<\omega$. On the other hand, the prefactor in the third term changes rapidly from 1 to 0 at $\omega_{L}=\omega$. With regard to $f_{r}\left(y_{-\omega+\gamma H}\right)$, it has two peaks at

$$
\omega_{L}=\omega_{ \pm}=\omega \pm \sqrt{\omega^{2}-\left(\gamma B_{1}\right)^{2}} .
$$

The peak at $\omega_{+}$is eliminated by the prefactor, while the peak at $\omega_{-}$, which behaves as $\left(\gamma B_{1}\right)^{2} / 2 \omega$ at small $B_{1}$, survives and defines the position of maximum in $R(B)$. For the two driving amplitudes plotted in Fig. 2 the expected shifts of the maxima are related as $9: 4$, which is indeed the case.

(ii) The Hanle curves broaden with increasing the drive amplitude. Formally, this follows from the broadening of the step-like behavior of the prefactor in the third term with $B_{1}$.

(iii) Upon increasing $B_{1}$, the Hanle curves exhibit signatures of magnetic resonance. True magnetic resonance, $\omega_{L}=\omega$, is certainly present only in the dynamics of $S_{z}$. In the dynamics of $S_{x}$ and $S_{y}$ the manifestations of 


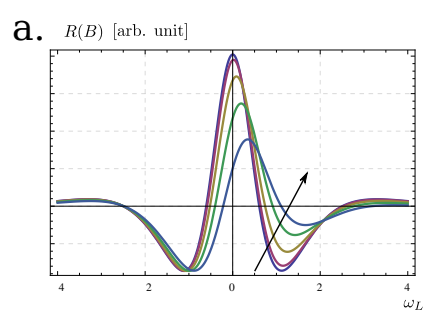

C. $\tilde{R}(B)[$ arb. unit $]$

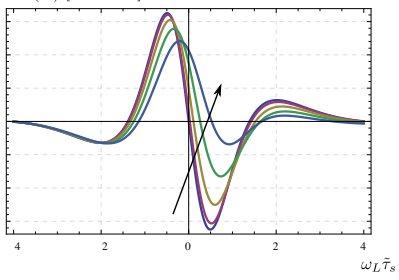

b. $R(B)$ [arb. unit $\rfloor$

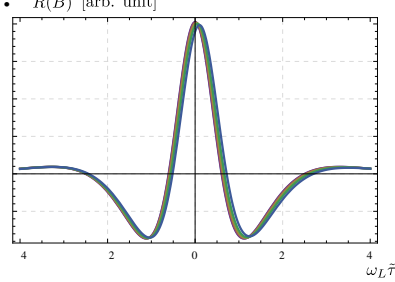

d.

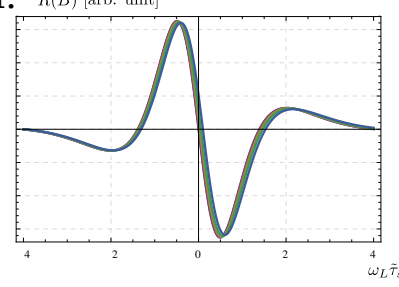

FIG. 3: [Color online] (a) In a spin-transport device with a long channel the prime effect of the ac drive on the Hanle curve is elimination of a minimum. The $R(B)$ curves are plotted from Eq. 14 for $L / \lambda_{s}=2.4, \omega \tilde{\tau}_{s}=1.1$ and five values: $0,0.25,0.5,0.75$, and 1 of the driving field, $\gamma B_{1} \tilde{\tau}_{s}$. Black arrow shows the direction in which $\gamma B_{1}$ increases. (b) Same curves as (a) but for the high-frequency drive, $\omega \tilde{\tau}_{s}=5$, show a weak response to the drive. (c) and (d) are the same as (a) and (b) for $\tilde{R}(B)$.

magnetic resonance is vague and originates from the fact that the derivative of the arguments $\omega \pm \gamma H$ with respect to $B$ is equal to $\left(\omega \pm \gamma B_{0}\right) / \gamma H$. It passes through zero at the magnetic-resonance condition and changes rapidly from -1 to 1 in its vicinity. This change translates into a kink-like behavior indicated in Fig. 2, More pronounced signatures of the magnetic resonance can be seen in the derivative $d R / d B$ also shown in Fig. 2, The derivative develops a plateau.

The shapes of $\tilde{R}(B)$ shown in Fig. 2 evolve with the drive in a predictable fashion. Namely, the position of zero shifts to finite magnetic field $\sim\left(\gamma B_{1}\right)^{2} / 2 \omega$ and the curves broaden. The signatures of magnetic resonance are more pronounced in $\tilde{R}(B)$. As seen in Fig. 2 the derivative $d \tilde{R} / d B$ exhibits a jump-like behavior near $\omega_{L}=\omega$.

\section{B. The injector and the detector are far apart}

In a long device, $\tilde{L} \gg 1$, the nonlocal resistance Eq. (5) exhibits oscillations decaying with magnetic field. First two zeros correspond to magnetic fields $\omega_{L} \tilde{\tau}_{s} \approx \pi / 2 \tilde{L}$ and $\omega_{L} \tilde{\tau}_{s} \approx 3 \pi / 2 \tilde{L}$. The effect of the ac drive on $R(B)$ is most pronounced when the driving frequency lies between these two values. This is illustrated in Fig. 3. Two sets of curves in Fig. 3 correspond to the same values of the drive amplitudes but to different driving frequencies. In the left and right sets the frequency differ by a factor of 5. It is seen that the Hanle shapes in the right set do not respond to the drive. The reason for that is

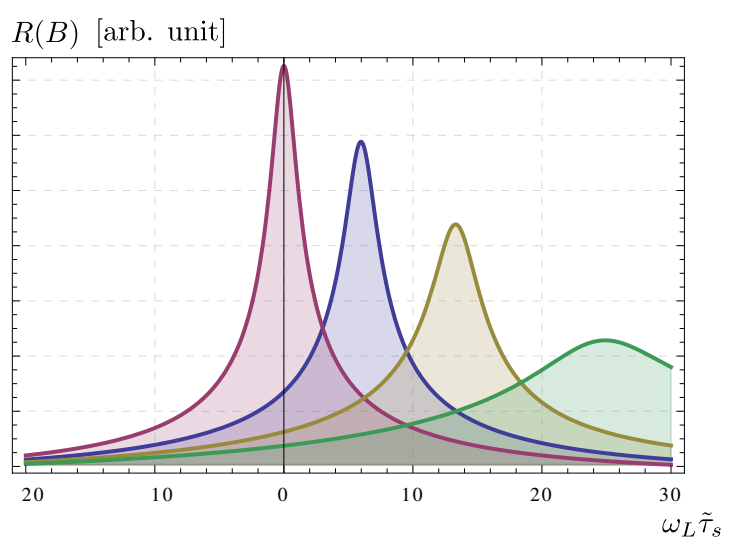

FIG. 4: [Color online] Evolution of the Hanle curves in a short device with increasing the amplitude of drive and very high driving frequency, $\omega \tilde{\tau}_{s}=30$, The curves are plotted from Eq. (14) for the values of $\gamma B_{1} \tilde{\tau}_{s}: 0$ (magenta), 18 (blue), 25 (tan), and 30 (green). The prime effect of drive is the shift of the maximum with slow broadening of the shape.

that the value $\omega \tilde{\tau}_{s}$ for this set is 5 , which is much bigger than $\pi / \tilde{L} \approx 1.4$. For the left set, $\omega \tilde{\tau}_{s}=1.1$, which is close to $\pi / \tilde{L}$. The lively response of $R(B)$ and $\tilde{R}(B)$ to the drive at this frequency originates from the fact that a non-driven curve is flat around $\omega_{L} \tilde{\tau}_{s} \approx 1.2$. With the choice $\omega \tilde{\tau}_{s}=1.1$ this $\omega_{L}$ is near magnetic resonance and fast change of the prefactors in Eq. (14) with $\omega_{L}$ is not overshadowed by the change of the function $f_{r}(y)$.

\section{LINEAR POLARIZATION OF THE DRIVE}

The expressions for nonlocal resistance obtained in the previous section are exact, in the sense, that they apply at arbitrary strengths and frequencies of the circularly polarized drive. We analyzed them for the situation when both $\gamma B_{1}$ and $\omega$ are comparable to the width of the Hanle curve. It is easy to see from Eq. (14) what happens to the Hanle curve when both $\gamma B_{1}$ and $\omega$ are much bigger than $\gamma B_{0}$. With the shape of the Hanle curve dominated by the third term in Eq. (14), the argument $-\omega+\gamma H$ of $f_{r}$ in this term can, at low $B_{0}$, be expanded as

$$
-\omega+\gamma H \approx-\omega+\left[\left(\gamma B_{1}\right)^{2}+\omega^{2}\right]^{1 / 2}-\frac{\gamma B_{0} \omega}{\left[\left(\gamma B_{1}\right)^{2}+\omega^{2}\right]^{1 / 2}} .
$$

Eq. (19) suggests that under a fast and strong circularlypolarized drive the Hanle curve simply shifts to the right preserving its shape. This is illustrated in Fig. 4 where the $R(B)$ curves are plotted from Eq. (14) for fast and strong drives. We intentionally chose a very high driving frequency $\omega \tilde{\tau}_{s}=30$ to allow the $R(B)$ peak to shift substantially with increasing $\gamma B_{1} \tilde{\tau}_{s}$.

Obviously, under a linearly polarized drive, the $R(B)-$ dependence maintains its symmetry with respect to 
$B=0$. From Fig. 4 one would expect that, when the maximum of $R(B)$ for circularly polarized drive is shifted by more than the width in the absence of drive, then the effect of linearly polarized drive would be a "symmetrized" peak. This is, actually, not the case. The reason is that the spin dynamics for a fast linearly polarized and circularly polarized drives are very different.

Assume that the driving field oscillates along the $x-$ axis, $\mathbf{B}_{1}(t)=\mathbf{i} B_{1} \cos (\omega t+\varphi)$. Then equations of motion for the spin projections assume the form

$$
\begin{aligned}
\frac{d S_{x}}{d t} & =-\gamma B_{0} S_{y}, \\
\frac{d S_{y}}{d t} & =\gamma B_{0} S_{x}-\gamma B_{1} S_{z} \cos \omega t, \\
\frac{d S_{z}}{d t} & =\gamma B_{1} S_{y} \cos \omega t .
\end{aligned}
$$

To handle the fast linearly polarized drive it is convenient ${ }^{32}$, to switch to the variables

$$
\begin{aligned}
& S_{x^{\prime}}=S_{x}, \\
& S_{y^{\prime}}=S_{y} \cos \theta(t)+S_{z} \sin \theta(t), \\
& S_{z^{\prime}}=-S_{y} \sin \theta(t)+S_{z} \cos \theta(t),
\end{aligned}
$$

where the angle $\theta(t)$ is defined as

$$
\theta(t)=\frac{\gamma B_{1}}{\omega} \sin (\omega t+\varphi)
$$

The physical meaning of the above transformation is moving into the frame rotating around the ac field; the ac field is "canceled" in the new frame. The equations of motion for the new variables read

$$
\begin{aligned}
\frac{d S_{x^{\prime}}}{d t} & =\gamma B_{0} S_{z^{\prime}} \sin \theta(t)-\gamma B_{0} S_{y^{\prime}} \cos \theta(t), \\
\frac{d S_{y^{\prime}}}{d t} & =\gamma B_{0} S_{x^{\prime}} \cos \theta(t), \\
\frac{d S_{z^{\prime}}}{d t} & =-\gamma B_{0} S_{x^{\prime}} \sin \theta(t) .
\end{aligned}
$$

One can see that there are two natural frequencies in the system Eq. (27), one is $\gamma B_{0}$ and the other is $\omega$. Since the second frequency is much bigger than the first, we can average the equations over time interval $\left(-\frac{\pi}{\omega}, \frac{\pi}{\omega}\right)$ assuming that the spin projections do not change significantly during this interval. Taking into account that $\langle\cos (\theta)\rangle=J_{0}\left(\gamma B_{1} / \omega\right)$, where $J_{0}(z)$ is a zero-order Bessel function, we get

$$
\begin{aligned}
\frac{d S_{x^{\prime}}}{d t} & =-\gamma B_{0} J_{0}\left(\frac{\gamma B_{1}}{\omega}\right) S_{y^{\prime}}, \\
\frac{d S_{y^{\prime}}}{d t} & =\gamma B_{0} J_{0}\left(\frac{\gamma B_{1}}{\omega}\right) S_{x^{\prime}}, \\
\frac{d S_{z^{\prime}}}{d t} & =0 .
\end{aligned}
$$

We see that the dynamics after averaging is slow, which justifies the averaging performed ${ }^{32}$. Upon returning to the lab frame the solution of the system Eq. 30 satisfying the condition $S_{x}(0)=1$ reads

$$
\begin{aligned}
& S_{x}(t)=\cos \left[\gamma B_{0} J_{0}\left(\frac{\gamma B_{1}}{\omega}\right) t\right], \\
& S_{y}(t)=\sin \left[\gamma B_{0} J_{0}\left(\frac{\gamma B_{1}}{\omega}\right) t\right] \cos \left[\frac{\gamma B_{1}}{\omega} \sin (\omega t+\varphi)\right], \\
& S_{z}(t)=\sin \left[\gamma B_{0} J_{0}\left(\frac{\gamma B_{1}}{\omega}\right) t\right] \sin \left[\frac{\gamma B_{1}}{\omega} \sin (\omega t+\varphi)\right] .
\end{aligned}
$$

As a final step, we average over the initial phase, $\varphi$, and obtain

$$
\begin{aligned}
& \left\langle S_{x}(t)\right\rangle=\cos \left[\gamma B_{0} J_{0}\left(\frac{\gamma B_{1}}{\omega}\right) t\right], \\
& \left\langle S_{y}(t)\right\rangle=J_{0}\left(\frac{\gamma B_{1}}{\omega}\right) \sin \left[\gamma B_{0} J_{0}\left(\frac{\gamma B_{1}}{\omega}\right) t\right], \\
& \left\langle S_{z}(t)\right\rangle=0 .
\end{aligned}
$$

The above result leads us to the conclusion that, with fast linearly polarized drive, the curves $R(B)$ and $\tilde{R}(B)$ have exactly the same shape as in the absence of drive. The only difference is that the Larmour frequency, $\omega_{L}$ gets replaced by $\omega_{L} J_{0}\left(\gamma B_{1} / \omega\right)$, signifying the broadening of the curves, which oscillates with the drive amplitude.

\section{A. Strong drive}

Another regime of the spin dynamics specific for a linearly polarized drive is realized when the drive is very strong, $B_{1} \gg B_{0}$. We will describe this regime qualitatively. As $\mathbf{B}_{1}(t)$ oscillates, it exceeds the static field $B_{0}$ during, practically, the entire period, $2 \pi / \omega$. Then $B_{0}$ has a negligible effect on the spin dynamics. However, during short time intervals, $\delta t$, when $\mathbf{B}_{1}(t)$ passes through zero, the electron spin is affected by $B_{0}$ only. During each of these intervals the spin rotates by the angle $\sim B_{0} \delta t$. Thus, the net rotation after time $t$ is $\sim\left(B_{0} \delta t\right) \omega t$. Now the value $\delta t$ can be estimated from the relation $B_{1}(\omega \delta t)=B_{0}$. This leads us to the conclusion that the spin dynamics, averaged over the the period of drive, is still a regular spin precession around the $z$-axis but with effective frequency $\sim \gamma B_{0}^{2} / B_{1}$ instead of $\omega_{L}$. One consequence of the replacement of $\omega_{L}$ by $\gamma B_{0}^{2} / B_{1} \ll \omega_{L}$ in Eq. (5) is a general broadening of the Hanle profile, which can be controlled by the strength of the drive. The other consequence is that the Hanle profile acquires a flat top.

\section{DISCUSSION}

- Our overall conclusion is that the ac drive with frequency, $\omega$, affects nonlocal spin transport if its am- 


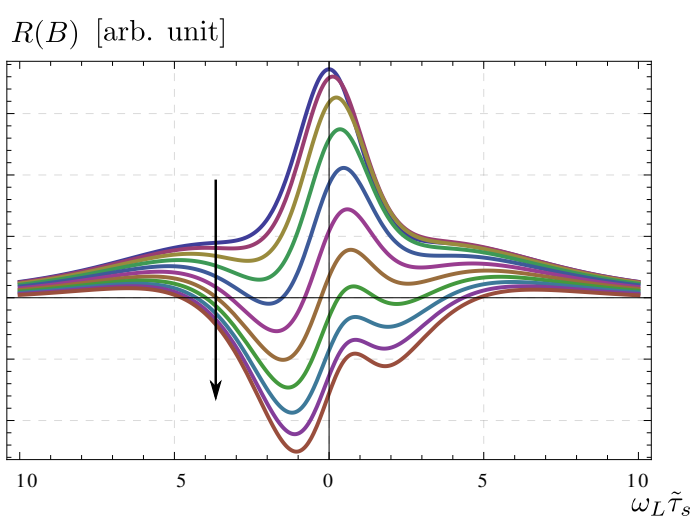

FIG. 5: At strong drive $\gamma B_{1}>\omega$. The zero-drive Hanle curves not only loose their symmetry but aquire additional maxima and minima. The evolution of the Hanle shapes for a given $\gamma B_{1} \tilde{\tau_{s}}=2$ and $L / \lambda_{s}=1.4$ is plotted from Eq. 14 for driving frequencies $\omega \tilde{\tau_{s}}$ taking the values between 0 and 1 with a step 0.1. Arrow shows the direction of the increase of the driving frequency.

plitude is strong enough, $\gamma B_{1} \gtrsim\left(\frac{\omega}{\tilde{\tau}_{s}}\right)^{-1 / 2}$. Choosing for an estimate the values, $\tau_{s}=50 \mathrm{~ns}$ and $\omega=200 \mathrm{MHz}$, we find that the driving amplitude must be bigger than $1 \mathrm{mT}$ to affect the spintransport. This value is quite realistic for experiments where the effects of the ac drive are studied by electrical measurements 33 .

- In this paper we considered the domain of parameters $\gamma B_{1}<\omega$ and found that modification of the shapes of the Hanle curves is primarily the broadening and the shift of the maximum. The above numerical estimate suggests that the opposite relation, $\gamma B_{1}>\omega$ is also experimentally accessible. For this domain of a strong circularly polarized drive the shapes of the Hanle curves change dramatically, as it is illustrated in Fig. 5. The curves exhibit two scales, which can be qualitatively interpreted as follows. The low- $B$ scale is the signature of the condition $\omega_{L}=\omega$ for which the argument $y_{-\omega+\gamma H}$ of $f_{r}$ in Eq. (14) is minimal. The high- $B$ feature is the signature of $\omega_{L}=\gamma B_{1}$ at which the prefactor in the third term in Eq. 14 changes significantly.

- One of our main findings is that the fast and strong circularly polarized ac field affect the Hanle pro- file dramatically, as illustrated in Fig. 4, while the linearly polarized field with the same amplitude and frequency has a little effect on the Hanle curve. This is in contrast to usual reasoning in magnetic resonance suggesting to treat the linear polarization as superposition of two circular polarizations and keep only the polarization which corotates with precessing electron spin. The easiest way to understand the difference is the spin dynamics for linear and circular polarizations qualitatively is to set $B_{0}$ equal to zero. Then, with initial spin direction along $x$, the ac field, linearly polarized along $x$, would not cause any spin dynamics at all. On the other hand, according to Eqs. (11) and (12), the average spin will precess around $z$-axis (if $\left.\gamma B_{1} \ll \omega\right)$ even when $B_{0}=0$.

- The effect of the ac drive on the Hanle curve is more pronounced for circular polarization of the drive when the symmetry of the curve is broken. In experiment, the circular polarization of microwaves is achieved $34 \sqrt[36]{3}$ with the help of two crossed microstrip resonators. In particular, in Ref. (35) it was demonstrated that ODMR spectrum of the nitrogen vacancies in diamond depends on the direction of the circular polarization of microwaves.

- Sensitivity of the Hanle curves to the ac-drive can serve as additional proof that a spin-polarized current indeed flows through the channel of the device. This proof is especially important for threeterminal devices where the question about the spin injection is still controversial $137 / 38$. In these devices, with only one of electrodes being a ferromagnet, the spin-dependent buildup of a voltage between injector and detector observed in experiment, and even sensitivity of this voltage to the magnetic field, ${ }^{8 / 37 / 38}$ can be caused by different delicate mechanisms. For example, a small inhomogeneity of the magnetization of the electrode might cause this sensitivity. Obviously, this source of magnetoresistance would not be sensitive to the ac-drive.

\section{ACKNOWLEDGEMENTS}

We are grateful to C. Boehme and H. Malissa for insightful discussions. This work was supported by NSF through MRSEC DMR-1121252.
1 F. J. Jedema, A. T. Filip, and B. J. van Wees, Nature (London) 410, 345 (2001).

2 M. Johnson and R. H. Silsbee, Phys. Lett. 55, 1790 (1985).

${ }^{3}$ M. Johnson and R. H. Silsbee, Phys. Rev. B 37, 5312 (1988).

4 I. Appelbaum, B. Huang, and D. Monsma, Nature (Lon- don) 447, 295 (2007).

5 B. Huang and I. Appelbaum, Phys. Rev. B 77, 165331 (2008).

6 J. Li, B. Huang, and I. Appelbaum, Appl. Phys. Lett. 92, 142507 (2008); B. Huang, H.-J. Jang, and I. Appelbaum, Appl. Phys. Lett. 93, 162508 (2008). 
7 T. Sasaki, T. Oikawa, T. Suzuki, M. Shiraishi, Y. Suzuki, and K. Noguchi, IEEE Trans. Magn., 46, 1436 (2010).

${ }^{8}$ Y. Aoki, M. Kameno, Y. Ando, E. Shikoh, Y. Suzuki, T. Shinjo, and M. Shiraishi, Phys. Rev. B 86, 081201(R) (2012).

${ }^{9}$ L.-T. Chang, W. Han, Y. Zhou, J. Tang, I. A. Fischer, M. Oehme, J. Schulze, R. K. Kawakami, and K. L. Wang, Semicond. Sci. Technol. 28, 015018 (2013).

${ }^{10}$ K. Kasahara, Y. Fujita, S. Yamada, K. Sawano, M. Miyao, and K. Hamaya, Appl. Phys. Express 7, 033002 (2014).

11 S. A. Crooker, M. Furis, X. Lou, C. Adelmann, D. L. Smith, C. J. Palmstrøm, and P. A. Crowell, Science 309, 2191 (2005).

12 X. Lou, C. Adelmann, S. A. Crooker, E. S. Garlid, J. Zhang, S. M. Reddy, S. D. Flexner, C. J. Palmstrøm, and P. A. Crowell, Nat. Phys. 3, 197 (2007).

${ }^{13}$ K. Olejník, J. Wunderlich, A. C. Irvine, R. P. Campion, V. P. Amin, J. Sinova, and T. Jungwirth, Phys. Rev. Lett. 109, 076601 (2012).

14 S. Majumder, B. Kardasz, G. Kirczenow, A. S. Thorpe, and K. L. Kavanagh, Semicond. Sci. Technol. 28, 035003 (2013).

15 J. Misuraca J.-I. Kim, J. Lu, K. Meng, L. Chen, X. Yu, J. Zhao, P. Xiong, and S. von Molnár, Appl. Phys. Lett. 104, 082405 (2014).

16 N. Tombros, C. Jozsa, M. Popinciuc, H. T. Jonkman, and B. J. van Wees, Nature (London) 448, 571 (2007).

17 C. Józsa, M. Popinciuc, N. Tombros, H. T. Jonkman, and B. J. van Wees, Phys. Rev. Lett. 100, 236603 (2008); Phys. Rev. B 79, 081402 (2009).

18 M. H. D. Guimarães, A. Veligura, P. J. Zomer, T. Maassen, I. J. Vera-Marun, N. Tombros, and B. J. van Wees, Nano Lett. 12, 3512 (2012).

19 T. Yamaguchi, Y. Inoue, S. Masubuchi, S. Morikawa, M. Onuki, K. Watanabe, T. Taniguchi, R. Moriya, and T. Machida, Appl. Phys. Express 6, 073001 (2013).

20 A. Dankert, M. V. Kamalakar, J. Bergsten, and S. P. Dash, Appl. Phys. Lett. 104, 192403 (2014).

21 E. S. Garlid, Q. O. Hu, M. K. Chan, C. J. Palmstrøm, and P. A. Crowell, Phys. Rev. Lett. 105, 156602 (2010).
22 S. Kuhlen, K. Schmalbuch, M. Hagedorn, P. Schlammes, M. Patt, M. Lepsa, G. Güntherodt, and B. Beschoten, Phys. Rev. Lett. 109, 146603 (2012).

23 S. Jahangir, F. DoGan, H. Kum, A. Manchon, and P. Bhattacharya, Phys. Rev. B 86, 035315 (2012).

24 M. Johnson and R. H. Silsbee, Phys. Rev. B 76, 153107 (2007)

25 Y. Niimi, Y. Kawanishi, D. H. Wei, C. Deranlot, H. X. Yang, M. Chshiev, T. Valet, A. Fert, and Y. Otani, Phys. Rev. Lett. 109, 156602 (2012).

26 Y. Niimi, H. Suzuki, Y. Kawanishi, Y. Omori, T. Valet, A. Fert, and Y. Otani, Phys. Rev. B 89, 054401 (2014).

27 S. Watanabe, K. Ando, K. Kang, S. Mooser, Y. Vaynzof, H. Kurebayashi, E. Saitoh, and H. Sirringhaus, Nat. Phys. 10, 308 (2014).

28 M. C. Prestgard and A. Tiwari, Appl. Phys. Lett. 104, $122402(2014)$.

29 Y. Manzke, R. Farshchi, P. Bruski, J. Herfort, and M. Ramsteiner, Phys. Rev. B 87, 134415 (2013).

30 G. Wang, B. L. Liu, A. Balocchi, P. Renucci, C. R. Zhu, T. Amand, C. Fontaine, and X. Marie, Nat. Commun. 4, 2372 (2013).

31 I. I. Rabi, Phys. Rev. 51, 652 (1937).

32 R. Glenn, M. E. Limes, B. Pankovich, B. Saam, and M. E. Raikh, Phys. Rev. B 87, 155128 (2013).

33 D. R. McCamey, K. J. van Schooten, W. J. Baker, S.-Y. Lee, S.-Y. Paik, J. M. Lupton, and C. Boehme, Phys. Rev. Lett. 104, 017601 (2010).

34 T. P. Mayer Alegre, A. C. Torrezan, and G. MedeirosRibeiro, Appl. Phys. Lett. 91, 204103 (2007).

35 T. P. Mayer Alegre, C. Santori, G. Medeiros-Ribeiro, and R. G. Beausoleil, Phys. Rev. B 76, 165205 (2007).

36 J. J. Henderson, C. M. Ramsey, H. M. Quddusi, and E. d. Barco, Rev. Sci. Instrum. 79, 074704 (2008).

37 S. J. Dash, S. Sharma, R. S. Patel, M. P. de Jong, and R. Jansen, Nature (London) 462, 491 (2009).

${ }^{38}$ K. R. Jeon, B. C. Min, I. J. Shin, C. Y. Park, H. S. Lee, Y. H. Jo, and S. C. Shin, Appl. Phys. Lett. 98, 262102 (2011). 